
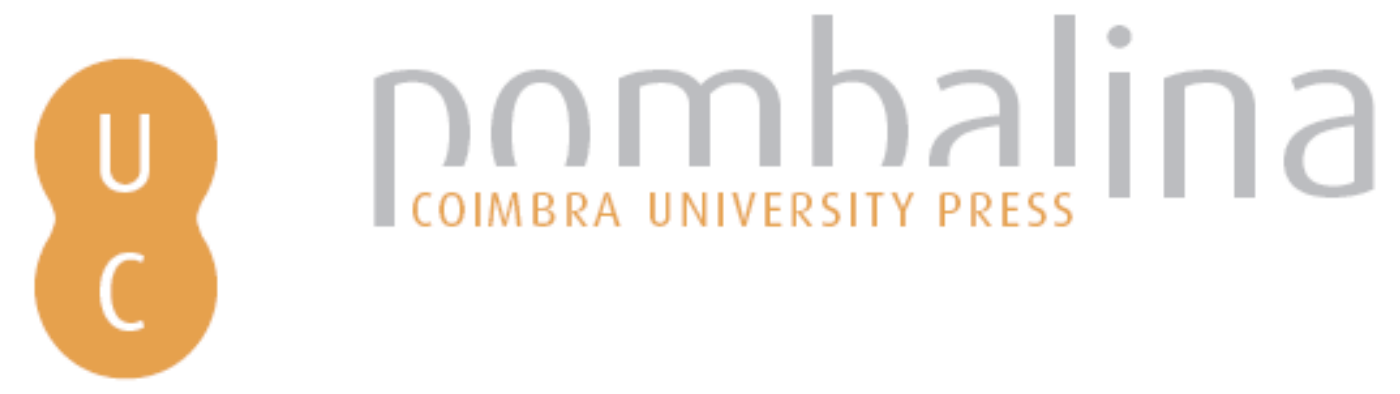

\title{
Características de la investigación Española en historia moderna a partir de las actas de congresos
}

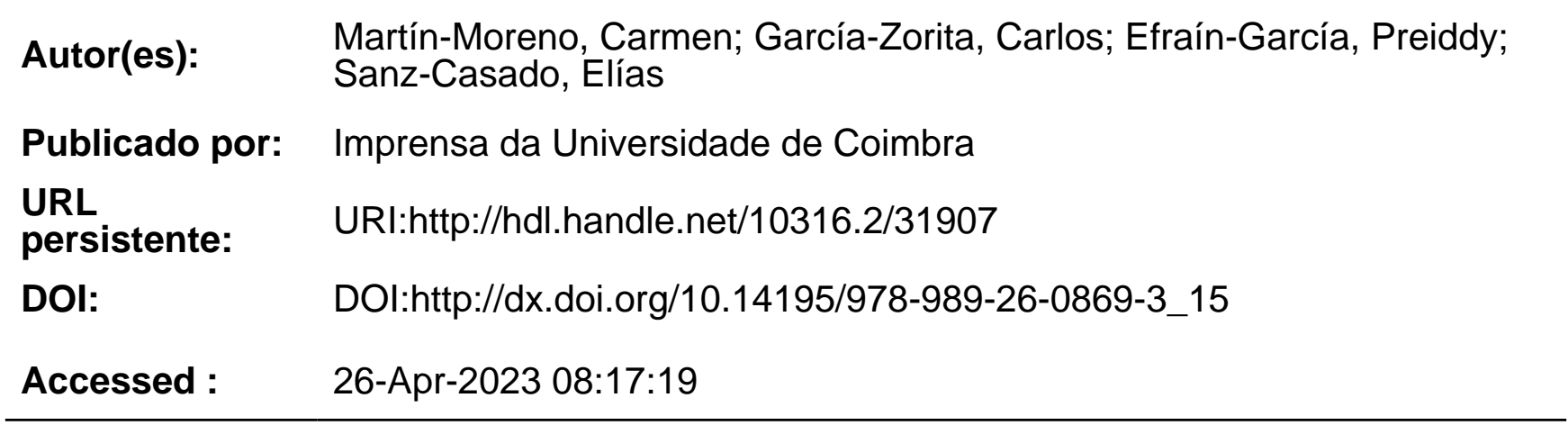

A navegação consulta e descarregamento dos títulos inseridos nas Bibliotecas Digitais UC Digitalis, UC Pombalina e UC Impactum, pressupõem a aceitação plena e sem reservas dos Termos e Condições de Uso destas Bibliotecas Digitais, disponíveis em https://digitalis.uc.pt/pt-pt/termos.

Conforme exposto nos referidos Termos e Condições de Uso, o descarregamento de títulos de acesso restrito requer uma licença válida de autorização devendo o utilizador aceder ao(s) documento(s) a partir de um endereço de IP da instituição detentora da supramencionada licença.

Ao utilizador é apenas permitido o descarregamento para uso pessoal, pelo que o emprego do(s) título(s) descarregado(s) para outro fim, designadamente comercial, carece de autorização do respetivo autor ou editor da obra.

Na medida em que todas as obras da UC Digitalis se encontram protegidas pelo Código do Direito de Autor e Direitos Conexos e demais legislação aplicável, toda a cópia, parcial ou total, deste documento, nos casos em que é legalmente admitida, deverá conter ou fazer-se acompanhar por este aviso.

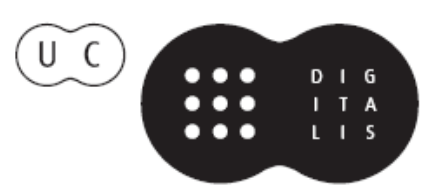


Maria Manuel Borges

Elias Sanz Casado

Coordenação

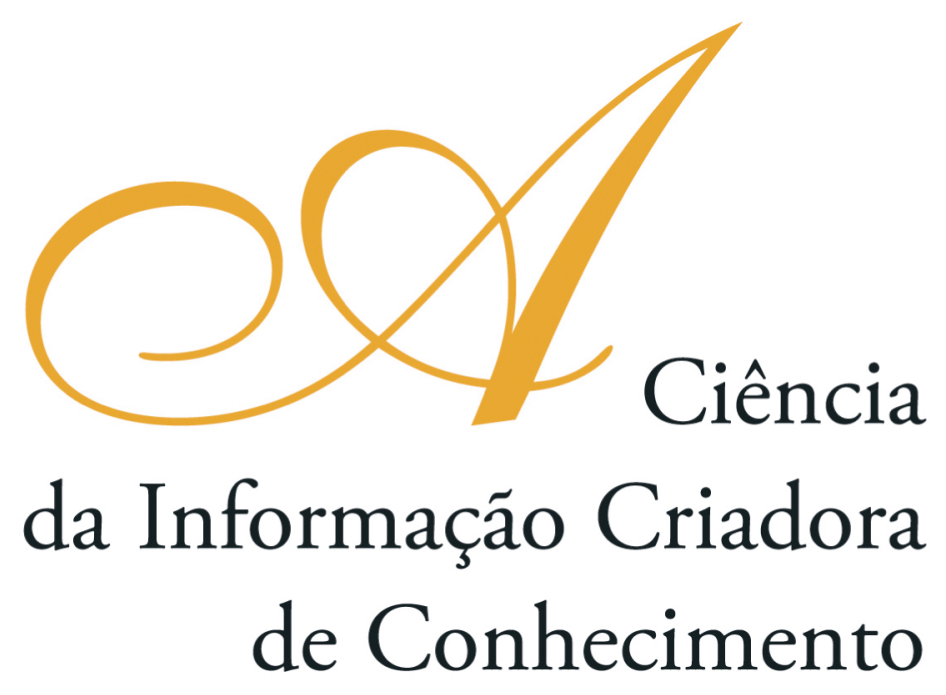

Vol. I I

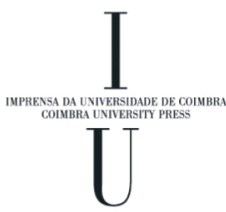

- COImbra 2009 


\title{
Características de la Investigación Española en Historia Moderna \\ A PARTIR DE LAS ACTAS DE CONGRESOS
}

\author{
Carmen Martín-Moreno \\ Universidad Carlos III de Madrid (Espanha) \\ Carlos García-Zorita \\ Universidad Carlos III de Madrid (Espanha) \\ Preiddy Efraín-García \\ Universidad Carlos III de Madrid (Espanha) \\ Elías Sanz-Casado \\ Universidad Carlos III de Madrid (Espanha)
}

\section{Resumen}

Se ha realizado un análisis bibliométrico de las actas de congresos y sus referencias bibliográficas, sobre Historia Moderna, recogidas en la base de datos Modernitas. Ésta es un índice de citas que recoge la producción científica española publicada en las revistas científicas españolas más prestigiosas, y en las actas de los congresos sobre Historia Moderna de mayor repercusión internacional. Esta base de datos recoge todas las referencias bibliográficas de los artículos y actas de congresos.

El trabajo ha permitido determinar algunas de las características más importantes sobre la investigación que han publicado estos autores, durante los años 2000 y 2001, así como sobre sus hábitos de consumo de información científica.

\section{Abstract}

A bibliometric analysis of the conference proceedings and their bibliography references included in Modernitas was made in the area of Modern History. Modernitas is a citation index that collects all Spanish scientific production published in more prestigious Spanish scientific journals and in conference proceedings about Modern History of great international impact. This database includes all bibliographic references of journal articles and conference proceedings.

This work has allowed determining some of the more important characteristics about the research published for these authors during the years 2000 and 2001, as well as of their habits of use of scientific information.

\section{Introducción}

La evaluación de la actividad investigadora es una actividad compleja que requiere abordajes desde distintas perspectivas, una de ellas es la de tipo cuantitativo. Esta perspectiva está experimentando desde hace varios años un fuerte desarrollo con la puesta a punto de una gran variedad y cantidad de metodologías y técnicas de trabajo, que están 
permitiendo relacionar múltiples aspectos vinculados con el sistema científico, como son las publicaciones, la colaboración científica o la antigüedad de las fuentes que consultan. Este tipo de evaluación se realiza a partir de la utilización de distintas metodologías, una de ellas, la bibliométrica, que implica el análisis de la producción científica de los investigadores, a partir de indicadores bibliométricos. Pese a que así se consigue una aproximación objetiva a una realidad tan compleja y variada como es la actividad investigadora, hay que reconocer que hasta el momento los estudios bibliométricos han demostrado una eficacia importante en determinados colectivos científicos, como son los de las ciencias puras, experimentales y tecnológicas, y en menor medida en las ciencias sociales. Sin embargo, los destinados a conocer la actividad científica de los investigadores en humanidades han sido muy escasos (Sanz Casado y Martín Moreno, 1997; Sanz Casado y otros, 1999). Esto ha sido debido, fundamentalmente, a la escasez de bases de datos que recogen exhaustivamente la investigación que publican los humanistas, preferentemente en monografías, revistas nacionales y actas e congresos, unas fuentes mal recogidas incluso por las bases de datos de la Plataforma Web of Science, orientadas a las humanidades. (Sanz Casado y otros, 2002)

Ante esta situación se decidió emprender un proyecto de investigación, con el fin de elaborar un instrumento adecuado para el análisis de la investigación en Historia Moderna espańola. Para ello, se decidió crear un índice de citas que recogiera la producción científica española publicada en las revistas científicas más prestigiosas, así como en actas de los congresos internacionales de mayor repercusión, celebrados en el período 2000-01.

El objetivo general de este estudio ha sido el de determinar las características de la investigación de los científicos españoles de Historia Moderna que publican sus resultados científicos en actas de congresos. Para ello, se ha analizado distintas características de las investigaciones que publican estos científicos, así como de la bibliografía que consultan como fundamento de dicha investigación.

\section{Metodología}

Este trabajo se ha centrado en la producción científica de los autores españoles de Historia Moderna que han publicado sus trabajos en las actas de los congresos celebrados en esta disciplina durante los años 2000 y 2001. También se analizaron las características de la investigación previa utilizada por los autores; para ello se extrajeron las referencias bibliográficas contenidas en las 756 actas correspondientes a 12 congresos distintos de Historia Moderna. El análisis de las características específicas de la investigación se ha determinado a partir de la obtención de una batería de indicadores bibliométricos. La elaboración de estos indicadores se ha llevado a cabo mediante técnicas estadísticas descriptivas.

\section{Resultados y discusión}

En primer lugar nos vamos a centrar en todos los resultados referidos a las actas de congresos fuente, para posteriormente pasar a los obtenidos con las referencias 
bibliográficas. El análisis se ha realizado sobre tres aspectos principales: distribución de los documentos publicados, idioma de los mismos, y colaboración entre autores.

\subsection{Resultados obtenidos de los documentos fuente}

En primer lugar se determinará como se distribuyen los documentos en las actas de congresos. Para ello, se analizará el número de trabajos que contiene las actas recogidas en cada uno de los 12 congresos considerados.

El número de trabajos publicados por cada documento fuente es muy variado, puesto que va desde las 142 actas que se han incluido en el congreso de Carlos V. Europeísmo y Universalidad, hasta las 16 en el de Isabel La Católica y la Política. El 51\% de la producción recogida en los doce congresos se encuentra en los cuatro primeros congresos. En la tabla 1 se recogen los títulos y el número de actas publicadas en cada uno de los 12 congresos analizados.

Tabla 1 - Actas publicadas en los 12 congresos seleccionados, en el periodo 2000-01

\begin{tabular}{|l|c|c|}
\hline Congresos & Actas & $\%$ \\
\hline Carlos V. Europeismo y Universalidad. Actas del co... & 142 & 18,86 \\
\hline Madrid, Felipe II y las ciudades de la Monarquía & 89 & 11,82 \\
\hline Carlos V y la quiebra del humanismo político en Eu... & 79 & 10,49 \\
\hline Congreso Internacional ‘El Conde de Aranda y su ti... & 74 & 9,83 \\
\hline El Emperador Carlos y su tiempo: actas IX Jornadas... & 72 & 9,56 \\
\hline De la unión de coronas al Imperio de Carlos V. Act... & 58 & 7,70 \\
\hline Las Órdenes Militares en la Península Ibérica & 52 & 6,91 \\
\hline Dinero, moneda y crédito en la Monarquía Hispánica... & 49 & 6,51 \\
\hline Andrés Laguna. Humanismo, ciencia y política en la... & 43 & 5,71 \\
\hline Las Universidades Hispánicas: de la Monarquía de l... & 43 & 5,71 \\
\hline El siglo de Carlos V y Felipe II. La construcción ... & 36 & 4,78 \\
\hline Isabel La Católica y la política & 16 & 2,12 \\
\hline Total actas & 753 & 100 \\
\hline
\end{tabular}

Otro de los aspectos que se estudió en estas fuentes, fue la capacidad idiomática de estos autores. Como puede observase en la tabla 2, hay grandes diferencias en el uso de los idiomas a la hora de redactar las actas de congresos. En este sentido, de las 753 actas analizadas, 696 de ellas (92,06\%), están escritas en castellano, 3,57\% en italiano, y el $1,32 \%$ en inglés y francés respectivamente. La presencia de idiomas extranjeros, podría deberse a que dichos congresos tienen carácter internacional, lo que supone la asistencia de historiadores de otros países que escriben los documentos en su lengua materna. 
Tabla 2 - Idioma de publicación de las actas de congresos

\begin{tabular}{|l|c|c|}
\hline Idioma & Actas & $\%$ \\
\hline Castellano & 694 & 92,16 \\
\hline Italiano & 26 & 3,45 \\
\hline Inglés & 10 & 1,33 \\
\hline Francés & 10 & 1,33 \\
\hline Portugués & 6 & 0,80 \\
\hline Catalán & 5 & 0,66 \\
\hline Alemán & 2 & 0,27 \\
\hline TOTAL & 753 & 100 \\
\hline
\end{tabular}

Respecto a la colaboración entre los autores, el índice de coautoría es de 1,07 autores por trabajo. Los 753 trabajos publicados como actas de congresos fueron firmados por 808 autores, de los que 705 trabajaron de manera individual. Esto viene a indicar que los historiadores espańoles analizados siguen patrones similares a los descritos anteriormente (Stone, 1982; Wiberley y Jones, 1994; Sanz y otros, 2002), colaborando para realizar distintos proyectos de investigación (Brockman y otros, 2001), pero escribiendo sus trabajos mayoritariamente de forma individual.

En cuanto a la productividad de los autores, hay que señalar que de los 813 autores diferentes, el 32,78\% de ellos ha publicado al menos dos trabajos. De los 114 autores que podríamos llamar más productivos, uno ha publicado 5 actas de congresos, 10 son autores de cuatro, 15 han escrito tres actas y 88 autores publicaron dos de sus trabajos como actas de congresos. En este caso, el indicador de Elite de Price tiene un valor de 28,51 .

\subsection{Resultados obtenidos a partir de las referencias bibliográficas}

Antes de comenzar el estudio de los distintos indicadores obtenidos de las referencias bibliográficas, hay que señalar que las 753 actas de congreso fuente contenían un total de 25.200 referencias.

En primer lugar se ha estudiado el tipo de documento que citan preferentemente los historiadores que han publicado en actas de congresos. En la tabla 3 se presentan los resultados del análisis sobre la tipología documental utilizada, y hay que señalar el gran uso que se hace de documentos con carácter monográfico, puesto que si se tienen en cuenta, además de las monografías propiamente dichas, las fuentes historiográficas y las obras colectivas, el porcentaje de este tipo de documentos se eleva hasta el 75,94\% del total. Estos datos tienen una gran coincidencia con los encontrados por Sanz y otros (2002) en su trabajo sobre las publicaciones de los historiadores en revistas españolas. En dicho trabajo estos autores encontraron que la utilización de documentos monográficos representaba el 74,14\%.

Los datos observados contradicen la afirmación de Brockman y colaboradores (2001), ya que estos autores afirman en su trabajo que es una simplificación asumir la preferencia de los humanistas por las monografías. Sin embargo, tanto en los estudios que hemos realizado nosotros (Sanz, et al., 2002), como en los de otros autores (Bebout 
y otros, 1975; Stone, 1982; Broadus, 1987) se ha observado dicha predilección. De hecho, en la tabla 4, puede observarse que pese a utilizar distintos tipos de documentos, el predominio en el consumo de monografías para obtener información es muy alto. Estos resultados responden a las características de este colectivo, definidas en los primeros trabajos de la década de los 70, para conocer los hábitos de información de los humanistas (Bebout y otros, 1975; Stone, 1982; Broadus, 1987), y en los que se incidía en su preferencia por la utilización de monografías frente a otro tipo de documentos. En cambio, nuestros datos presentan ciertas discrepancias con los resultados de Knievel y Kellsey (2005), probablemente por los criterios que utilizaron los autores en el análisis de las referencias.

En cuanto al porcentaje de uso de los artículos de revista, el encontrado en este trabajo es inferior al observado por Sanz y otros (2002) en su trabajo sobre historia, ya que ellos observaron que los historiadores que publicaban en revistas españolas, el $21,9 \%$ de sus referencias bibliográficas correspondía a artículos de revista, frente al $16,21 \%$ que encontramos en este trabajo.

Tabla 3 - Tipo de documentos referenciados en las actas de congresos.

\begin{tabular}{|l|c|c|}
\hline Tipo Documental & Frecuencia & $\%$ \\
\hline Monografía & 12.035 & 47,76 \\
\hline Artículos de revista & 4.084 & 16,21 \\
\hline Fuentes historiográficas & 4.003 & 15,88 \\
\hline Obras colectivas & 3.099 & 12,30 \\
\hline Actas de congresos & 1.463 & 5,81 \\
\hline Tesis & 203 & 0,81 \\
\hline Otros & 313 & 1,24 \\
\hline Total & 25.200 & 100 \\
\hline
\end{tabular}

Otro de los aspectos que se quiso analizar fue la capacidad idiomática de los autores, a partir de determinar el idioma en que estaban escritas las referencias consultadas en los documentos fuente. En la tabla 4 se recogen los nueve idiomas que tienen una mayor presencia en las referencias de actas fuente, encontrándose ordenados de mayor a menor en función de la frecuencia con que aparecen en las referencias.

Las 25.200 referencias bibliográficas se encontraban escritas en 16 idiomas distintos, si bien en la tabla 4 solo se recogen los 9 que aparecen con mayor frecuencia, y el resto se incluye en la categoría de Otros, que recoge idiomas como el gallego, polaco, turco, etc. El idioma principal, con un $69,76 \%$ \%, es el castellano. Esto es lógico dado que la mayoría de los autores de las actas de congresos fuente, tienen el castellano como lengua materna. Los otros dos idiomas predominantes son el italiano, que representa el 7,36\%, y del francés, con un porcentaje similar (7,28\%).

Una conclusión que puede sacarse es que cerca del $30 \%$ de las referencias de las actas, se hicieron a trabajos en lenguas extranjeras, un porcentaje mucho mayor que el 19,5\% encontrado por Knievel y Kelsey (2005), y que está en consonancia con la observada en múltiples trabajos anteriores, gran capacidad idiomática de los humanistas (Bebout y otros, 1975; Stieg, 1981), y que está relacionada con su necesidad investigadora de consultar documentos originales y no traducidos. 
Tabla 4 - Idiomas de las referencias en actas de congresos.

\begin{tabular}{|l|c|c|}
\hline Idioma & Frecuencia & $\%$ \\
\hline Español & 17.578 & 69,75 \\
\hline Inglés & 1.433 & 5,69 \\
\hline Francés & 1.835 & 7,28 \\
\hline Catalán & 551 & 2,18 \\
\hline Latín & 519 & 2,06 \\
\hline Italiano & 1.854 & 7,36 \\
\hline Alemán & 860 & 3,41 \\
\hline Portugués & 240 & 0,95 \\
\hline Holandés & 271 & 1,08 \\
\hline Otros & 59 & 0,32 \\
\hline TOTAL & 25.200 & 100 \\
\hline
\end{tabular}

Otro aspecto de interés fue el determinar la obsolescencia de la literatura utilizada por este tipo de historiadores. Para ello se han calculado anualmente los dos indicadores que permiten conocer dicha característica, la Vida Media (VM) y el Índice de Price (IP). Una vez determinados estos valores para los dos ańos estudiados, se calculó el valor medio de cada uno de ellos y que hemos denominado VMM e IPM. Los valores encontrados han sido de 18,69 ańos de VMM y el 14,63\% de IPM, para los dos ańos estudiados. Estos datos son muy similares a los encontrados en otros trabajos que estudiaron este tipo de investigadores (Sanz y col., 2002).

En cuanto a la colaboración observada en los autores responsables de las referencias bibliográficas, hay que señalar que el índice de coautoría es de 1,06 autores por documento. El número de autores distintos a los que se ha referenciado ha sido de 9.715. Como puede observarse, el valor del índice de coautoría de las referencias es muy similar al obtenido para los trabajos fuente $(1,07)$, lo cual viene a corroborar la escasa colaboración que se produce en general en las publicaciones de esta comunidad científica.

Con respecto a la dispersión de las revistas más consultadas por los autores de actas de congresos, hay que señalar que los más de cuatro mil artículos de revista referenciados en la bibliografía se encontraban recogidos en 1.198 títulos, de los cuales, sólo 692 habían publicado un artículo. El núcleo estaría conformado por 108 revistas, que son con las que estos científicos cubren más del $50 \%$ de sus necesidades de información. La representación gráfica de dicho indicador se muestra en la Fig. 1. 


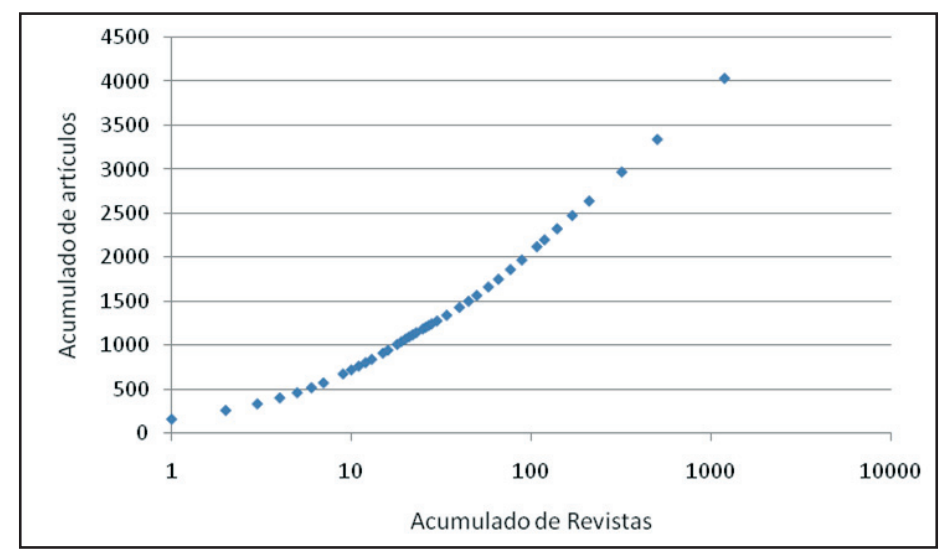

Fig. 1 - Dispersión de las revistas citadas en las actas de congresos fuente.

Si consideramos solo aquellas revistas que fueron referenciadas al menos 30 veces, encontramos los 19 títulos que se muestran en la tabla 5. Entre ellas, las 5 que cuentan con un mayor número de referencias son: Hispania. Revista española de Historia con el 4,09\% de las referencias, Anuario de Historia del Derecho Español, con el 2,5\%, Boletín de la Real Academia de la Historia con el 1,84\%, Cuadernos de Historia Moderna con el 1,69\%, y Bulletin Hispanique con el 1,46\%.

Tabla 5 - Revistas más citadas por los autores de actas fuente

\begin{tabular}{|l|c|c|}
\hline Títulos de revista citados en actas de congresos & Frecuencia & $\%$ \\
\hline Hispania. Revista espańola de Historia & 165 & 4,09 \\
\hline Anuario de Historia del Derecho Espańol & 100 & 2,5 \\
\hline Boletín de la Real Academia de la Historia & 74 & 1,84 \\
\hline Cuadernos de Historia Moderna & 68 & 1,69 \\
\hline Bulletin Hispanique & 59 & 1,46 \\
\hline Anuario de Estudios Americanos & 56 & 1,39 \\
\hline Studia Historica. Historia Moderna & 55 & 1,26 \\
\hline $\begin{array}{l}\text { Archivo Ibero-americano. Estudios históricos sobre la Orden Franciscana } \\
\text { en Espaná y sus misiones }\end{array}$ & 51 & 1,26 \\
\hline Revista de Archivos, Bibliotecas y Museos & 51 & 1,26 \\
\hline Chronica Nova & 45 & 1.12 \\
\hline Manuscrits. Revista d'Història Moderna & 43 & 1,07 \\
\hline Hidalguía & 39 & 0,97 \\
\hline Mélanges de la Casa de Velázquez & 38 & 0,94 \\
\hline Anuario de Estudios Medievales & 35 & 0,87 \\
\hline Estudis. Revista d'Història Moderna & 35 & 0,87 \\
\hline Baetica. Estudios de Arte, Geografía e Historia & 34 & 0,84 \\
\hline En la Espańa Medieval & 33 & 0,82 \\
\hline Moneda y Crédito. Revista de Economía & 33 & 0,82 \\
\hline Hispania Sacra & 31 & 0,77 \\
\hline Total & 1.045 & 24,72 \\
\hline
\end{tabular}


En el caso de las referencias que los autores de actas de congresos fuente realizan a otros congresos, hemos encontrado que son solo 8 los congresos que aparecen referenciados más de 20 veces (tabla 6). Los 3 congresos que aparecen con mayor frecuencia son: Felipe II y el Mediterráneo con el 3,30\% de las referencias, Las sociedades ibéricas y el mar a finales del siglo XVI con el 2,23\%, y Felipe II (1527-1598): Europa y la monarquía católica con el $2,16 \%$.

Tabla 6 - Actas más citadas por los autores de actas fuente.

\begin{tabular}{|l|c|c|}
\hline Actas de congresos más citadas & No Doc & $\%$ \\
\hline Felipe II y el Mediterráneo & 46 & 3,3 \\
\hline Las sociedades ibéricas y el mar a finales del siglo XVI & 31 & 2,23 \\
\hline Felipe II (1527-1598): Europa y la monarquía católica & 30 & 2,16 \\
\hline Congreso Internacional de Historia: 'El Tratado de Tordesillas y su época & 26 & 1,87 \\
\hline Seńorío y Feudalismo en la Península Ibérica (siglos XII-XIX) & 24 & 1,72 \\
\hline XV Congreso de Historia de la Corona de Aragón. Siglos XV-XVI & 24 & 1,72 \\
\hline $\begin{array}{l}\text { Congreso Internacional Las órdenes militares en el Mediterráneo occidental (siglos } \\
\text { XII al XVIII) }\end{array}$ & 22 & 158 \\
\hline $\begin{array}{l}\text { Congreso Internacional de Historia de las Universidades Americanas y Espańolas } \\
\text { en la Edad Moderna: Claustros y estudiantes }\end{array}$ & 21 & 1,51 \\
\hline Total & 224 & 16,1 \\
\hline
\end{tabular}

\section{Conclusiones}

Se ha observado una gran productividad en 4 de los 12 congresos analizados, puesto que sólo ellos son responsables del $51 \%$ de las actas. Concretamente los más productivos han sido el de Carlos V. Europeísmo y Universalidad con 142 actas, seguido de Madrid, Felipe II y las ciudades de la Monarquía con 89 actas, el de Carlos V y la quiebra del humanismo politico en Europa con 79 actas, y el Congreso Internacional "El Conde de Aranda y su tiempo" con 74 actas.

En cuanto a los idiomas en los que se han publicado las actas de congresos, la mayoría han sido escritas en castellano (el 92,06\%). Los otros 3 idiomas más frecuentes han sido el italiano (3,57\%), y el inglés y francés con el ,32\% cada uno de ellos.

La colaboración establecida por los autores de las actas de congresos es muy escasa, apenas 1,07 autores por documento. Esto viene a confirmar el patrón de trabajo individual que aún siguen manteniendo estos investigadores, y que ya ha sido observado en trabajos anteriores, aunque en la mayoría de los casos a partir del análisis de revistas o monografías.

Cuando se analiza la bibliografía referenciada en las actas de congresos, se observa que el tipo de documento que citan mayoritariamente son los de carácter monográfico $(75,94 \%)$. Esto también viene a corroborar la preferencia de estos autores por este tipo de fuente, independientemente de dónde se publique la investigación que realizan. También ésta es una característica observada desde los primeros estudios realizados sobre este tipo de científicos. 
La revista científica es la segunda fuente más utilizada, aunque con un valor porcentual muy alejado de la monografía.

Otro aspecto analizado fue la capacidad idiomática a la hora de la utilización de documentos. En este sentido, se observó un amplio rango de idiomas en los que se encontraba la información consultada, aunque el que aparecía con un mayor porcentaje fue el castellano, seguido del italiano y el francés. Aunque los valores de estos dos últimos idiomas eran notablemente inferiores al primero de ellos.

La obsolescencia de la información utilizada también estuvo entre los rangos ya observados para la investigación en este tipo de disciplinas, con una gran cantidad de material de tipo clásico, que es la que justifica una Vida Media elevada de 18,69 años, y un Índice de Price bajo, concretamente del 14,63\%.

La colaboración observada en las referencias bibliográficas, muestra el mismo esquema de colaboración establecida por los autores fuente y a la observada en trabajos anteriores; es decir, estos investigadores trabajan fundamentalmente de forma individual.

\section{Referências bibliográficas}

Bebout, L.; Davis, D. \& Oehlerts, D. (1975). User studies in the humanities: a survey and a proposal. $R Q, 15$ (1): 40-44.

Broadus, R. N. (1987). Information needs of humanities scholars: A study of request made at the National Humanities Center. Library and Information Science Research, 9 (2): 113-29.

Brockman, W. S y otros. (2001). Scholarly work in the humanities and the evolving information environment. Washington, D. C.: Digital Library Federation; council on Library and Information Resources.

Knievel, J. E.; Kellsey, C. (2005). Citation analysis for collection development: a comparative study of eight humanities fields. Libr Q. 75 (2):142-68.

Sanz Casado, E.; Martín Moreno, C. (1997). Técnicas bibliométricas aplicadas a los estudios de usuarios. Revista General de Información y Documentación, 7 (2): 41-68.

Sanz Casado, E.; y otros. (1999). La investigación española en Economía a través de las publicaciones nacionales e internacionales en el período 1990-1995. Revista de Economía Aplicada, 7 (20): 113-37.

Sanz, E.; Castro, F.; Povedano, E.; Hernández, A.; Martín, C.; Morillo-Velarde, J.; García-Zorita, C.; De la Nuez, J. L.; Fuentes, M. J. (2002). Creación de un índice de citas de revistas españolas de Humanidades para el estudio de la actividad investigadora de los científicos de estas disciplinas. Revista Española de Documentación Científica. 25 (4): 443-454.

Stieg, M. F. The information needs of historians. College \& Research Libraries, 1981, 42 (6): 549-61.

Stone, S. (1982). Humanities scholars: information needs and uses. Journal of Documentation, 38 (4): 292-313.

Wiberley, S. E.; Jones, W. G. (1994). Humanists revisited: a longitudinal look at the adoption of information technology. College \& Research Libraries, 55: 499-509. 